\title{
How Valuable Is It?
}

\section{Henrik Andersson ${ }^{1} \cdot$ Jakob Green Werkmäster ${ }^{1}$ (D)}

Published online: 1 July 2020

(c) The Author(s) 2020

\section{Introduction}

An acceptable analysis of value should not only account for what it is for an object to be valuable, it should also be able to account for how valuable it is. ${ }^{1}$ While work in value theory usually focuses on the first desideratum, the second is unfortunately often neglected. This is true for one of the main analyses of value in the current philosophical debate, the Fitting-Attitudes analysis of value (henceforth the FA analysis). ${ }^{2}$ The FA analysis provides an account of an object being valuable in terms of

\footnotetext{
1 Our usage of "object" is regrettably a bit idiosyncratic, by object we refer to anything that can be the bearer of value. What exactly can function as the bearer of value, such as states of affairs, concrete objects, and so forth is controversial and something we wish to remain neutral on, hence the usage of 'object'.

2 The FA analysis has a long history see Frans Brentano, The Origin of Our Knowledge of Right and Wrong, trans. R. M. Chisholm, (London, Routledge \& Kegan Paul, 1889/1969). For a historical overview see Wlodek Rabinowicz and Toni Rønnow-Rasmussen, "The Strike of the Demon: On Fitting Pro-attitudes and Value," Ethics 113(3) (2004): 391-423. It is also often referred to as the Buck-Passing Analysis of value after T.M Scanlon What We Owe to Each Other, (Cambridge: Belknap, 1998). In the literature, there are different ways to distinguish between the FA analysis and the Buck-Passing analysis of value. While there are interesting distinctions to be made between the two, these differences are of no relevance for the purposes of our paper. For the sake of clarity, we will only be referring to this line of analysis as the "FA analysis". For a discussion on this see Conor McHugh \& Jonathan Way, "Fittingness First" Ethics 126(3) (2016): 575-606; Richard Rowland, The Normative and the Evaluative: The Buck-Passing Account of Value, (Oxford: Oxford University Press, 2019); Mark Schroeder "Value and the right kind of reason," Oxford Studies in Metaethics, vol 5 (2010): 25-55; Jussi Suikkanen, "Buck-passing acounts of value," Philosophy Compass 4(5) (2009): 768-799.
}

Henrik Andersson and Jakob Green Werkmäster have contributed equally and are listed alphabetically.

Jakob Green Werkmäster

Jakob.green_werkmaster@fil.lu.se

Henrik Andersson

Henrik.andersson@fil.lu.se

1 Department of Philosophy, Lund University, Lund, Sweden 
it being fitting to direct pro-attitudes or con-attitudes towards said object. Its generic formulation has the following form:

FA analysis generic form: $x$ is valuable if and only if it is fitting to favor $x .^{3}$

The "fitting"-part of the analysis (henceforth the "normative component") is often, but by no means always, spelled out in terms of reasons. ${ }^{4}$ The "favor"-part of the analysis is a placeholder for any type of pro-attitude (or in the case of negative value, a con-attitude) such as admiring, loving, appreciating, and is what we will call the "attitudinal component" of the FA analysis.

Despite the FA analysis' popularity, not much has been written on how we are to account for how valuable an object is. That is, most accounts settle for an analysis of, for example, what it is for an object to be good rather than bad, but do not account for what it is for an object to be, for example, very good rather than moderately good. Such accounts provide only half the picture of "value" when they fail to provide an account of degrees of value. Note that it is not only that the interest of a complete account has the virtue of satisfying the curiosity of value theorists. It can also be claimed that while it is helpful to know that two options are good, it may be even more helpful to know how good the options are in order to make an informed decision. Consequently, the notion of "how valuable" something is seems to play an important role in our everyday life. Furthermore, if it turns out that the FA analysis cannot provide a satisfactory account of degrees of value this should make us hesitant to accept the FA analysis because any satisfactory account of value ought to be able to account for degrees of value. ${ }^{6}$ Thus, in order for the FA analysis to be a serious alternative, this needs to be rectified. The aim of this paper is to provide

\footnotetext{
3 There is a vast amount of issues concerning how we are to understand the FA analysis, such as e.g., whether the analysis is reductive or not, or whether it should be understood as circular or not see Rabinowicz \& Rønnow-Rasmussen, op. cit., and W.D Ross Foundations of Ethics, (Oxford: Oxford University Press, 1969/1939). We believe that nothing that we say hinges on one's stance on these other issues regarding the FA analysis. Not to mention the vast array of objections that has been put forward towards the FA analysis in general such as the Wrong Kind of Reasons problem (for a recent overview see Jan Gertken \& Benjamin Kiesewetter, "The right and the wrong kind of reasons," Philosophy Compass 12(5) (2017)) or how it handles solitary goods (see Krister Bykvist, "No Good Fit: Why the Fitting Attitude Analysis of Value Fails," Mind 118(469) (2009): 1-30) to name a few. While interesting, these objections are beyond the scope of this paper.

4 See Ulrike Heuer, "Beyond Wrong Reasons: The Buck-Passing Account of Value," in M. Brady (ed.), New Waves in Metaethics (London: Palgrave Macmillan UK, 2011), 166-184; Pamela Hieronymi, "The Wrong Kind of Reason," Journal of Philosophy, 102(9), (2005): 437 - 457; Rabinowicz \& RønnowRasmussen, op. cit.; Schroeder op. cit.

5 There is a debate on whether the FA analysis should be understood to be a semantic thesis about what it means to be valuable or as a metaphysical thesis on what it is to be valuable. In this paper we endorse it as a metaphysical thesis, and we will have reason to discuss this further later on in the paper. Furthermore, we use the more neutral 'account for' rather than for example 'determine', 'explain', or 'ground'. This is because what kind of relationship it actually is depends on how one view the metaphysical relationship between value and reasons. For more on this see Rowland, op. cit.

${ }^{6}$ We are grateful to an anonymous referee for pressing us to clarify what is at stake and for suggesting that the viability of the FA analysis is one important part of the answer.
} 
an exploratory investigation and hopefully spur a fruitful discussion. Since it is an undertheorized topic, we put an emphasis on outlining the different positions rather than settling the question on which position is ultimately correct. That being said, we of course argue in favor of the alternative that we believe has the most potential.

In the paper, we present two different strategies for developing the FA analysis so that it is able to account for of how good something is. The first strategy focuses on the normative component in the analysans while the second strategy focuses on the attitudinal component in the analysans. After a thorough exploration of the two strategies we conclude that the latter strategy is more fruitful.

\section{View One: The Normative Component View}

Consider the view that it is only the strength of the normative component that accounts for how good something is. We will refer to this position as the Normative Component View. One might find this position intuitive - if the reasons for favoring $x$ and the reasons for favoring $y$ differ significantly in strength this should somehow be reflected in their values.

The claim that it is the normative component that should play the central role can be found as early as in G. E. Moore's work. In his review of Franz Brentano's The Origin of the Knowledge of Right and Wrong Moore criticized Brentano for understanding "better" in terms of "worthy of more love" rather than "more worthy of love" 7 . He supported this possibility by not only referring to the intuitive appeal we highlighted above but also by providing additional considerations. He writes:

[Brentano's] first suggestion is that since "good" means "worthy to be loved," "better" must mean "worthy of more love". It does not seem to have occurred to him that it must mean "more worthy of love," that is to say, his attention is directed only to that element of his definition, which is a "concrete physical content," namely the love, not the more important element "rightness," which is not. In asserting that a thing is rightly loved with a greater love, you do not assert that it possesses the quality of being rightly loved in any higher degree than what is rightly loved with a less love; hence if good means rightly loved, you do not assert that it is better. ${ }^{8}$

We take this quote to highlight two interesting observations. The first is the rather straightforward structural claim; if we are to make a comparative out of "worthy to be loved" it is not the content, i.e., "to be loved" that is to be formed into comparative form, but rather the "worthiness." The second observation is that Moore takes it to be the case that in a normative analysis it is the normative component that should

\footnotetext{
7 G. E. Moore, "Book Review: The Origin of Knowledge of Right and Wrong. Franz Brentano," Journal of Ethics 14(1) (1903): 115-123.

8 Moore, op. cit., p 21.
} 
do the normative heavy work. We take these observations to give some prima facie intuitive support for the Normative Component View. In other words, if something is more valuable this must be reflected in the normative component of the analysis. How valuable $x$ is, is accounted for by how fitting it is to favor $x$.

It is important to separate two different views of how to understand "fitting" when we are discussing the Normative Component View. For our present purposes there are two main families of interpretations of the FA analysis, one that understands the normative component in a non-gradable manner, and one that understands the normative component in a gradable manner. ${ }^{9}$ Each of these families of interpretations has their own sub-classes of interpretations. For brevity's sake we do not go into detail in to all the non-gradable readings, since the only thing that matters for this paper is that they all employ a non-gradable normative notion. An example of a non-gradable normative notion is that of a conclusive reason, and we understand an agent having a conclusive reason to favor $x$ if and only if the agent has stronger reason to favor $x$ than to not favor $x$.

We have divided the family of gradable interpretations of the FA analysis into two camps. First, the Pro Tanto Reading, which roughly says that an attitude is fitting to have towards an object if and only if there is a pro tanto reason to have the attitude, and secondly the Overall Reading, which roughly says that an attitude is fitting to have towards an object if and only if the overall balance of reasons favors having the attitude. The phrasing of overall balance of reasons might sound too similar to that of a conclusive reason, but there is an important difference - remember the concept is non-gradable. Something cannot be more or less a conclusive reason, either it is or it is not a conclusive reason. The overall balance of reason, however, can be more or less heavily in favor of one option rather than the other. To illustrate the difference between gradable notions of reasons and non-gradable notions of reasons, we can think of Usain Bolt winning a 100m race. The property of winning the race does not come in degrees, but of course he ran the race with a certain degree of speed in

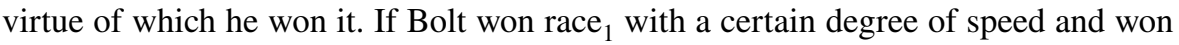
race $_{2}$ with a slower speed, this does not mean that he won the first race more than he won the second race. He won both races equally. Likewise, just because a conclusive reason would not have been conclusive in different circumstances, this does not mean that the reason is conclusive to a higher or lower degree. It is non-gradable.

\subsection{The Non-Gradable Interpretation}

Let us begin by showing how, rather obviously, a non-gradable interpretation is not compatible with the Normative Component View. Non-gradable interpretations can be traced back to an early formulation of the FA analysis that was championed by Franz Brentano. Brentano claimed that an object, $x$, is valuable if and only if love

\footnotetext{
9 Note that by "gradable" we do not mean that it is possible to determine the exact strength rather we treat it as a synonym to "comes in degrees".
} 
towards $x$ is "correct" (richtig). ${ }^{10}$ Among more contemporary commentators we find that "correct" has been substituted with "sufficient reason" or with "conclusive reason," or "ought"11 All of these different views have, for our purposes, one salient feature in common, i.e., they are all formulated in terms of a non-gradable normative component. Due to the non-gradable normative component it should be obvious that it is not compatible with the Normative Component View because the Normative Component View requires a gradable normative component to get off the ground. If the non-gradable interpretation is correct, then Normative Component View is implausible. On the other hand, if one finds the Normative Component View to be intuitively appealing, then this could be taken to count against the nongradable interpretation.

\subsection{The Gradable Interpretation}

It seems as if the Pro Tanto Reading of the FA analysis is straightforwardly compatible with the Normative Component View, albeit not in any way committed to it. In order for the Normative Component View to make sense, the normative component must be able to come in degrees, and pro tanto reasons is a bona fide example of something normative that comes in degrees, namely degrees of strength. On this view, if we have strong pro tanto reasons to favor $x$, then $x$ has a high value. If we have strong pro tanto reasons to favor $x$ and only weak pro tanto reasons to favor $y$, then $x$ is more valuable than $y$. How much more valuable $x$ is than $y$ is accounted for by the difference in strength between the pro tanto reasons to favor $x$ and the pro tanto reasons to favor $y$.

This simple picture of the Pro Tanto Reading, however, needs to become a bit more sophisticated. An object can be valuable in certain respects but also all-thingsconsidered valuable. ${ }^{12}$ For instance, global warming might be good in respect to wine production in northern Europe, but bad overall. If the improved prospect for wine production in northern Europe is a pro tanto reason to favor global warming it seems as the initial formulation of the Pro Tanto Reading entails that global warming is good. Likewise, if the prospect of millions of people dying due to global warming is a pro tanto reason to disfavor global warming then global warming is bad. Global warming therefore turns out to be both good and bad at the same time. Furthermore, since it is both good and bad, global warming turns out to be better than itself. The claim that global warming is both good and bad and better than itself might not seem that problematic; as the analysis is formulated above it doesn't state

\footnotetext{
10 See Brentano op. cit.

11 For "sufficient reason" see Gertken \& Kiesewetter, op. cit., and Schroeder, op. cit. For "conclusive reasons", or "ought" see A.C Ewin, The Definition of Good, (Westport Conn: Hyperion Press, 1948) and Wlodek Rabinowicz, "Value relations," Theoria 74(1) (2008): 18-49. While Kiesewetter and Gertken formulate themselves in terms of sufficient reason they do not provide an argument, nor are they, in their paper, committed to formulating it in terms of sufficient reason. For this reason, it might seem unfair to ascribe them this position. However, Gertken has in personal communication told us that they do so in order to avoid over flooding the world with too many values.

12 Some may prefer to call it "good period", "good simpliciter" or "overall value".
} 
whether it is an analysis of all-things-considered goodness or a more fine-grained notion of goodness. Consequently it can be argued that the above reasoning does not entail that global warming is all-things-considered bad and all-things-considered good but rather it entails that global warming is bad in some respects and good in other respects. We are hesitant to this suggestion since one would expect the more interesting FA analysis to be an analysis of "value period" and not only "value in a respect" and this suggestion seems to commit us to the claim that the Pro Tanto Reading can only give us an analysis of "good in a respect." It can also be shown that this line of reasoning comes with other problems.

First of all, even if we admit that things can be good in a respect this is not sufficient to avoid the objection. That is, for some valuable respects the same sort of objection that was presented above can be iterated. Let us illustrate with an example. Global warming is good with respect to wine production in northern Europe and bad with respect to melting ice caps. However, note that there very well could be a reason against bringing about global warming that relates to wine production in northern Europe. In order to determinately know that there are no such reasons the adherents of the Pro Tanto Reading must claim that if there is a pro tanto reason to favor (or disfavor) an object with regards to a certain respect there can be no other pro tanto reasons to disfavor (or favor) that object with regards to the same respect, nor can there be any other pro tanto reasons to favor (or disfavor) that object that is more or less strong than the initial pro tanto reason. This claim should be hard to accept since it seems central for the concept of "pro tanto reasons" that when there is such a reason in favor of ying there could be a contrasting reason against $y$ ing. ${ }^{13}$

The natural solution to the dilemma is the idea that what determines the value of an object are not the individual pro tanto reasons, but rather the balance of reasons. It is not fitting to favor global warming just because there are some pro tanto reasons to favor it, rather whether it is fitting or not depends on the strength of the pro tanto reasons taken together. This solution, however, is tantamount to abandoning the Pro Tanto Reading and instead opting for some sort of Overall Reading. This view roughly says that there may be pro tanto reasons to favor $x$ and pro tanto reasons against favoring $x$ and $x$ is only positively valuable if the overall balance of reasons favors favoring $x$. To illustrate, this would mean that global warming is all-thingsconsidered valuable if and only if the overall balance of reasons favors favoring global warming, and global warming is valuable with respect to wine production in northern Europe if and only if the overall balance of reasons favors favoring global warming with respect to wine production in northern Europe. How valuable it is is analyzed by the balance of reasons.

As previously argued, an FA analysis in terms of non-gradable concepts such as "sufficient reason" and "conclusive reason" is a non-starter. It is perhaps possible to

\footnotetext{
13 On one of the more influential views on the strength of reasons (Scanlon, 2014) it makes no sense to talk of a reason in isolation having any strength at all, because they are essentially comparative, i.e., reason can only be stronger, weaker, or equally as strong (except in matters of incommensurability) than another reason. Reasons are not simply "strong" or "weak". Does it make sense to claim that wine production in northern Europe is a strong pro tanto reason to favor global warming if it is, not even in principle, possible for there to be another pro tanto reason that it is stronger than?
} 
reconstrue these views in a manner similar to the above, making room for gradable concepts. However, such a view would thus end up being an Overall Reading and not a strict non-gradable reading. ${ }^{14}$

It is now clear what kind of position one should hold if one wishes to argue for the Normative Component View. The Normative Component View is incompatible with a reading of the FA analysis that treats the normative component as non-gradable, and yet a formulation in terms of the gradable notion of pro tanto reasons seems to have unwanted consequences. The problems can, however, be avoided if we formulate the FA analysis in terms of what we call the Overall Reading. With the Overall Reading a single object would not turn out to be both good and bad, nor would it be both very valuable and somewhat valuable.

From this we submit that the Normative Component View is more reasonable given the Overall Reading. That is, the Normative Component View is not a plausible view for those who endorse a non-gradable reading of the FA analysis. ${ }^{15}$ While we have no great qualms against the Overall Reading as a reading of the FA analysis, it is a disadvantage for the Normative Component View that it is committed to a specific reading of the FA analysis. Especially if one takes into account that, to our knowledge, very few, if any, have in text endorsed an FA analysis in terms of the overall balance of reasons. ${ }^{16}$

The Attitudinal Component View, on the other hand, as we will show, is not committed to a specific interpretation of the normative component. Given that there is no consensus in the literature on how to interpret the normative component it seems ceteris paribus that this neutrality tentatively is a theoretical virtue that speaks in favor of the Attitudinal Component View.

\footnotetext{
${ }^{14}$ If non-gradable normative concepts are reducible to gradable concept then of course the distinction between non-gradable and gradable interpretations of the normative component view collapses. This, however, does not affect our conclusion that the Normative Component View needs a gradable normative component. We are very grateful to an anonymous reviewer for forcing us to think twice about this line of reasoning.

15 Interestingly enough, it seems as if Richard Rowland (see op. cit) has recently defended the idea of understanding degrees of value via a combination of the Normative Component View and a FA analysis in terms of sufficient reason. He writes "And these different degrees of $X$ 's goodness and value correspond to the strength of the reasons that there are to respond to $X$ " (Rowland, op. cit., p. 14). This quote seems to be an endorsement of the Normative Component View. He goes on to define all-things-considered goodness (that he calls good overall) as follows: "Good Overall. What it is for $X$ to be non-instrumentally good simpliciter overall is just for there to be sufficient reason for us to have a non-instrumental pro-attitude towards $X$ " (Rowland, op. cit., p. 15). His understanding of sufficient reason is, however, not necessarily the same as ours and perhaps it is more charitable to ascribe him a gradable interpretation of reasons. We are grateful to an anonymous reviewer for pressing us on this.

${ }^{16}$ Wlodek Rabinowicz has in personal communication suggested that a problem with the Normative Component View is that it would make hard cases of comparing different kinds of value too easy. If only the degree of strength determined degrees of value, then it seems we could easily compare the aesthetic value of a painting and the moral value of being helped by a stranger by comparing how fitting it is to aesthetically admire the painting with how fitting it is to feel gratitude towards the person. We are unsure if we are swayed by this argument but we do think it is indeed a challenge for proponents of the Normative Component View.
} 
Before we compare the Normative Component View with its rival the Attitudinal Component View we first need to properly introduce the Attitudinal Component View.

\section{View Two: The Attitudinal Component View}

According to The Attitudinal Component View the amount of value an object has is to be accounted for in terms of the intensity of the attitude that it is fitting to have towards the object. ${ }^{17}$ This seems to be a widely accepted view, but seldom explicated or argued for. ${ }^{18}$ It is, however, easy to see why it is the preferred view. Assume that you have reasons with some specific strength to favor $x$ with a certain intensity and you have reasons with the same strength to favor $y$ but to a higher degree of intensity then one would think that this is indicative of some differences in value. ${ }^{19}$ That is, it may seem intuitively appealing that how strongly we feel for something reflects how valuable it is.

One of the more convincing lines of thought in favor of the Attitudinal Component View is the generic formulation of the FA analysis itself. We have focused on the question of how valuable an object is and mostly discussed the amount of value an object has but of course a part of answering how valuable an object is consists of determining whether it has a positive or a negative value. This is, according to all formulations of the FA analysis, accounted for by the valence of the attitudes. If it is fitting to favor an object then the object is good, while if it is fitting to disfavor it then the object is bad. In other words, all adherents of the FA analysis seem to believe that when accounting for how valuable an object is - as in "is it good or bad?" - one must look into what kind of attitude is fitting to have.

The fact that this valence is accounted for by the attitudinal component lends support to the claim that all statements regarding how good an object is ought to be accounted for by referring to the attitudinal component. ${ }^{20}$ It also strongly supports

\footnotetext{
17 From now on we will once again use the term 'fitting' as a placeholder for the normative component of the FA analysis.

18 See Rabinowicz, 2008 op. cit.; Schroeder, op. cit.; and John Skorupski, The Domain of Reasons, (Oxford: Oxford University Press, 2010) p. 86.

19 We here assume that if you, for instance, admire an object more intensely than another object then you admire the latter object more.

20 As an anonymous referee pointed out to us this does not rule out the Normative Component View. Since proponents of this view could claim that the valence of the value is determined by the valence of the attitude, but that the degree of the value is determined by the strength of the normative component. It should be noted that the solution suggested is in strict terms not compatible with the Normative Component View since the solutions entails that the part of how valuable something is determined by the attitudinal component and consequently, we end up with a hybrid view. A view that cannot be accused of being a hybrid version ought instead to be formulated in terms of the valence of the reasons. That is, something is bad if and only if there is a conclusive reason against favoring it. This formulation, however, entails the absurd conclusion that if the valence of the reason is positive then the object always has a value with a positive valence, e.g., the absurd conclusion that if there is a conclusive reason to disfavor an object the object will be positively valuable. This leaves us with the hybrid formulation. While this formulation may be coherent, we believe that the Attitudinal Component View is preferable. The Attitudinal Component View has the theoretical virtue of being simpler and more unified than the hybrid
} 
the claim that the attitudinal component plays some role in accounting for how valuable an object is, and is therefore a forceful objection against the Normative Component View (which assumes that the attitudinal component plays no role in accounting for how valuable an object is).

\subsection{Intensity of Attitudes}

There is of course a lot to be said about how to understand attitudes, what they are, and how to understand the intensity of an attitude. One way would be gauge the phenomenological experience of what it is "felt like." 21 Another way would be to understand it in functional or dispositional terms. Hate, for instance, not only seems to have a certain feel, but is also associated with other characteristics such as a tendency to attack, confront, or be filled with a blinding rage. The more nuanced details in how to understand the intensity of an attitude need not be our main concern; for our present purposes it suffices that we have some sort of intuitive grasp of having different attitudes to a higher or lesser degree of intensity. ${ }^{22}$

If we, for reasons of presentation, formulate the intensity of attitudes in terms of the phenomenological experience of what it is like-ness of having an episode of that attitude we can grade the intensity of attitudes on a one-dimensional scale. This has the advantage that the intensity of different attitudes can be placed on the very same scale in terms of their intensity, ranging from lower to higher intensity. ${ }^{23}$

It is essential for some attitudes to fall within a certain range on the intensity scale. It seems, again, central for hatred to be quite intense, even low-intense hatred is still quite intense. If it lacked sufficient intensity, we would not experience hatred but rather dislike or something of that kind.

Some attitudes may have a large range, perhaps even covering the entirety of intensity scale. This might be the case for an attitude such as favoring. ${ }^{24}$ It must also be made clear that pro-attitudes may overlap con-attitudes in terms of their intensity range, love and hatred may for example have the same lower- and upper bounds

\footnotetext{
Footnote 20 (continued)

formulation since in the former the attitude does all explanatory work. We will, however, consider hybrid formulations later on. Hopefully, that discussion will provide further reasons as to why hybrid formulations should be avoided.

${ }^{21}$ Julien Deonna, \& Fabrice Teroni, The Emotions: A Philosophical Introduction. (New York: Routledge, 2011) p. 1.

22 We do not want to claim that all emotions necessarily are pro- or con-attitudes nor do we want to claim that all pro- or con-attitudes necessarily are emotions, however, for an illuminating discussion about the intensity of emotions see Aaron Ben-Ze'ev, "Emotional Intensity," Theory \& Psychology 6(3) (1996): 509-532 and Christine Tappolet, Emotions, Values, and Agency (Oxford: Oxford University Press, 2016).

23 Note, however, that it is not necessarily the case that all attitudes can be placed on the same scale. That is, we do not endorse a kind of value monism. It is fully possible that there is a plurality of values and that some of these cannot be compared to each other. The Attitudinal Component View leaves room for such a possibility, by allowing for certain types of attitudes to not be placed on the same scale.

24 At least this must be the case for the adherents of the FA analysis that takes favoring to not only be a placeholder, but also an evaluative attitude on its own, i.e., as a pro-attitude able cover the entire scale of intensity.
} 
intensity wise. The intensity does not take into account the valence of the attitude. The Attitudinal Component View claims that it is the intensity, in this wide sense, of the attitude that captures the amount of value an object has. In other words, by knowing that it is fitting to favor $x$ we know that $x$ is valuable, and by knowing with what degree of intensity it is fitting to favor $x$ we know how valuable $x$ is.

It should also be noted that we have mentioned a complex version of the Attitudinal Component View. A much simpler version holds that value ought to be analyzed in a single attitude, e.g., desire. If this view is correct then there is no need to compare the intensity of different attitudes and the attitudinal view would be a rather straightforward analysis.

\subsection{Some Problems with the Attitudinal Component View}

It could be argued that certain objects may be very valuable, yet it is impossible to have the corresponding attitude with such great degree of intensity. The Attitudinal Component View cannot account for such a case. Note that the problem is not that it may be psychologically impossible to have such an intense feeling of admiration - the FA analysis is silent on what is psychologically possible - rather it is the fact that it seems to be conceptually impossible given the characterization above. The possible lack of symmetry between the intensity of the attitude and the amount of value could thus give rise to a conceptual hurdle for the Attitudinal Component View. However, in order for this objection to get off the ground we need plausible examples that establishes that it is possible that there are objects with a certain degree of value for which the corresponding attitude cannot be felt with the correct intensity. Possible examples might be God, humanity, or the good will, all of which are examples of something that might be claimed to be of infinite value. While we are suspicious towards the notion of infinite value in the first place, we still want to push back on the notion that if something has an infinite value then this should entail that we should, let's say, admire it with an infinite intensity. We fail to see why an infinite value would need to correspond to an infinite intensity of attitudes, rather, than perhaps the maximal intensity. Furthermore, if one is of the view that there are infinitely valuable objects then perhaps this could be captured by the idea that it is always fitting to, e.g., love God more than what one does. In this way, we are able to capture an object being infinitely valuable by it being the case that it is always fitting to have adopt a more intense attitude than what one currently has. ${ }^{25}$

Another potential objection is that we make too strong assumptions in characterizing The Attitudinal Component. Let us clarify. If the FA analysis is spelled out in the following way:

\footnotetext{
${ }^{25}$ See Brentano, op, cit., p. 104 and Michael Zimmerman, The Nature of Intrinsic Value, (Lanham, Maryland: Rowman \& Littlefield, 2001). Thanks to Jonas Olson for pushing us on the force of the objection from infinitely valuable objects.
} 
Generic FA analysis: $x$ is valuable if and only if it is fitting to favor $x$.

From this it follows that if it is fitting to favor $x$ then $x$ is valuable. Furthermore, if we make the reasonable assumption that anything that has a value, has a certain amount of that value, it follows that $x$ has a certain amount of value. ${ }^{26}$

This means, according to the Attitudinal Component View, that if it is fitting to favor $x$ it is fitting to do so with a certain intensity. Reasons for attitudes are thus, strictly speaking, reasons to have a certain attitude to a specific degree of intensity. It might seem odd that it cannot be the case that it is fitting to favor something without it being fitting to favor it with a certain degree of intensity. We do not, however, take this to be an objection to the Attitudinal Component View; it might just as well be seen as a remarkable result that follows if we accept the most reasonable interpretations of the FA analysis. The fact that this feature has not been discussed by others, may be due to the fact that previous work on the FA analysis has focused on the question of how to understand what it is, or means, for $x$ to have value, and in order to answer this question there is no need to specify any degree of intensity of attitudes; it is enough to say that an object is valuable if and only if it is fitting to favor it. ${ }^{27}$

This connects to another potential problem with the Attitudinal Component View. The proposed view seems to be incompatible with a wider understanding of the FA analysis that includes not only pro-attitudes but also pro-responses construed more broadly. Examples of such responses include actions, such as e.g., preserving an object. Since actions do not come in degrees, it seems odd to claim that it is intensity of the pro-response that corresponds to the amount of value the object has. One cannot preserve, promote, or protect something more or less intensely. On the other hand, it seems intuitive to say that certain objects are fitting to preserve. We see two possible ways to accommodate this intuition while remaining a proponent of the FA analysis and the Attitudinal Component View. The first possibility is to interpret "being fitting to preserve" in a less literal way. For example, one could take it to mean: "being fitting to have a disposition to preserve." ${ }^{28}$ Dispositions can be more or less strong. The second possibility is to assume some type of bridge-principle that links reasons for certain attitudes to reasons for certain actions. An example of such a principle is John Skorupski's Bridge principle: "Whatever facts give $x$ reason to

\footnotetext{
${ }^{26}$ Even though we take this assumption to be reasonable it can be questioned. The claim that everything that has value has a certain amount of value, seems to be incompatible with the claim that there can be incommensurability in the axiological domain. This assumption must then be tested against convincing arguments that shows that it is possible for two value bearers to not be related by an "at least as good as" relation. For the most developed argument see Ruth Chang, Making Comparisons Count, (London: Routledge, 2002). If it is true that not all value bearers are related by an "at least as good as" relation it seems as if the assumption must be rejected and consequently the objection presented above can be rejected. We do, however, find the assumption to be intuitive and do not want to accept the existence of incommensurability. However, we acknowledge that sometimes our value judgment cannot be precise enough to specify a certain specific amount of value, for instance if we are dealing with cases of vagueness where it is indeterminable exactly how valuable an object is, but determinable that the object is within a certain range of value.

27 The only exception we have found who acknowledges this possibility is Skorupski, op. cit., p 51, 86.

28 An alternative interpretation that might be more controversial, but similar in spirit, is that the reason to, for example, preserve, protect and promote are actually reasons to try to preserve, protect and promote. Trying to protect might be something that one can do with various degrees of intensity.
} 
feel $\varphi$ give $x$ a reason to do the $\varphi$-prompted action, in virtue of being a reason to feel $\varphi$." 29 This principle gives us the means to explain why certain objects are fitting to preserve without thereby saying that they are valuable in virtue of it being fitting to preserve: It is fitting to feel a pro-attitude towards an object, and in virtue of this it is fitting to do the action prompted by said attitude. It being valuable is, strictly speaking, understood purely in terms of fitting attitudes, but there is still a close connection to the fittingness of other pro-responses.

There is perhaps a temptation to opt for a hybrid view. On the hybrid view, how valuable an object is, is determined both by the strength of the normative component and the intensity of the attitudinal component. We advise against going this route. A hybrid view seems to retain all of the problems of the original views without retaining their simplicity. In addition, the hybrid view has its own set of problems. For instance, if both the normative component and the attitudinal component play $a$ role in determining how valuable an object is it is still clouded in mystery exactly what role that is. The hybrid view cries out for a function that tells how the two components together account for the exact value of an object. This function could take many different forms but it is hard to see how one would go about to argue for one formulation rather than the other. Should the strengths be added together, multiplied, or is it a more complex function? Finding leverage for one function rather than another seems like an impossible task. ${ }^{30}$

\subsection{Moore's objection}

Let us once again consider Moore's objection and compare the Normative Component View and the Attitudinal Component View. We took Moore to highlight two things. First, the structural claim: If "valuable" means "worthy of love" "more valuable" should mean "more worthy of love." Secondly, an intuition that it is the normative component that ought to do the explanatory work of accounting for how valuable something is. These two issues can easily be addressed. Let us begin with the first issue. The structural claim seems to be based on the linguistic intuition that the comparative form of a predicate $F$ is " $F$ er than" or "more $F$ than" consequently the

\footnotetext{
29 John Skorupski, “The triplism of practical reason," Ratio, 25(2) (2012): 127-147.

30 A possibility here is to insist there will be no problematic weighings, because the strength of the normative component and the strength of the attitudinal component supervenes on each other. On this view, it would of course be confused, or at least impossible, to say that there could be a case where we have strong normative component and a weak attitudinal component, or vice versa. This sort of connection, however, seems unreasonably ad hoc. Another possibility is that both the normative and the attitudinal component plays a role in determining the how valuable something is, however, they do so independently and not in the interdependent way sketched above. Roughly, the idea is that amounts of value is not linear (see e.g., Chang op. cit., and Rabinowicz, 2008 op. cit). This way the two components can model different dimensions of value. Even though this view may have the advantage of providing us with a big conceptual space for degrees of value; allowing for a multitude of ways in which values can relate to each other. In Rabinowicz, op. cit., for instance, there are fifteen different value relations. On the account currently under consideration the plurality of possible value relations would be greatly increased. Perhaps this plurality is in itself a reason to reject this view. After all, we would prefer to have an account that gives us the correct amount of value relations, rather than an account that allows us to model a dizzying amount of possible value relations.
} 
comparative form of "worthy of love" ought to be "more worthy of love." Indeed, as has been pointed out by Bykvist this principle of compositionality is quite reasonable. $^{31}$ Therefore, the Attitudinal Component View would potentially be in a lot of trouble if one assumes that the FA analysis is a semantic theory about what it means for something to be good, i.e., the view that "good" is synonymous with "worthy of love." Luckily, however, we are not advancing the FA analysis as a semantic theory, but rather an ontological theory about what it is for an object to be good, i.e. the metaphysical explanation of an object being good is that it is worthy of love. ${ }^{32}$ Because of this, Moore's argument that the Attitudinal Component View would violate some semantic principle of compositionality is beside the point.

Moore's second, and more worrying argument, is that if something has more value, then surely this must be reflected in the normative component of the analysis; the normative seems after all to be central for values. In other words it may seem confused to claim that an object is more valuable and thereby has a stronger normative pull, and yet the analysans fails to capture this. We find this line of response to be harder to refute. We can note, however, that we are in good company when we put forth an analysis that downplays this alleged connection between the evaluative and the normative. Consider for example standard FA analyses of different kinds of value:

Final Value: An object has final value if and only if it is fitting to favor an object for its own sake. ${ }^{33}$

Instrumental Value: An object has instrumental value if and only if it is fitting to favor it for the sake of its effects. ${ }^{34}$

Personal Value: An object is good for $\mathrm{P}$ if and only if it is fitting to favor the object for P's sake. ${ }^{35}$

Attributive Value: An object is good as an F if and only if it is fitting to favor the object in so far as one wants an F. ${ }^{36}$

All of these are similar in that they employ the concept of fittingness. What is being modified in these different analyses is not the normative component but the attitudinal component. Consider, for example, the analysis of instrumental value. It can be questioned whether this is a value at all, it is only a means to something that is valuable, yet the normative component is the same as the normative component in the analysans of final value. Surely Moore's objection should apply here as well: that an object has final value rather than instrumental must be reflected in the normative component of the analysis, by potentially introducing final reasons to favor and instrumental reasons to favor.

\footnotetext{
${ }^{31}$ Krister Bykvist, "Brentano's fallacy: Moorean arguments against Brentano-style fitting attitude analyses of value," Manuscript.

${ }^{32}$ Thanks to Wlodek Rabinowicz for helpful discussions about this topic.

${ }^{33}$ See Rabinowicz \& Rønnow-Rasmussen, op. cit., Zimmerman, op. cit.

${ }^{34}$ Ibid.

${ }^{35}$ See Toni Rønnow-Rasmussen, Personal Value, (Oxford: Oxford University Press, 2011)

${ }^{36}$ Alex Gregory, "A very good reason to reject the buck-passing account," Australasian Journal of Philosophy 92(2) (2014): 287-303.
} 
From this we submit that if Moore's objection is to be taken seriously, it should not only be construed as an objection to the Attitudinal Component View, but rather as an objection against most attempts to extend the generic FA analysis to also apply to different kinds of value. We take it, however, that most proponents of the FA analysis do not find Moore's objection too worrying and indeed accept analyses of different kinds of value such as those above. It does seem unfair to claim that the normative component must do all the heavy lifting in the analysis, the analysans does after all have two parts: a normative component and an attitudinal component. ${ }^{37}$ Indeed looking at contemporary attempts within in the FA analysis tradition of accounting for various different kinds of values in terms of modifying the attitudinal component rather than the normative component shifts the burden of proof. It establishes some sort of precedent and presumption in favor of the Attitudinal Component View rather than the Normative Component View. If the Normative Component View is correct in this instance, then be this would reason to doubt the prior analyses of e.g., final and instrumental value. However, the outlooks of understanding final value in terms of e.g., final reasons rather than favoring objects for their own sake look quite bleak.

\subsection{The Resilience of Value}

We should not, however, dismiss the intuition that the strength of the normative component plays some role in accounting for the value of the object. Indeed, it is quite persuasive. We share this intuition, and instead of dismissing it we want to propose a different explanation for what role the normative component plays when it comes to the value of object. We suggest that the strength of the normative component accounts for the resilience of the object's value. The resilience of an object's value is a dimension of value that has been overlooked in the current debate. This is unfortunate since conceptualizing it may be helpful when trying to get a fuller grasp of the domain of value. ${ }^{38}$ This notion merits further investigations but we can give

\footnotetext{
37 In this paper we have only focused on the intensity of the attitudes, but not mentioned the role of the length of the attitude that we have reasons to have. It could be suggested that how good something is, is reflected in how long it is fitting to have the attitude. If it is fitting to favor something for a long period then the object is very valuable, while if it is fitting to favor it for a brief period then the object is not as valuable. Even if this suggestion has some prima facie plausibility, we believe that the fitting longevity of attitudes does not account for how valuable an object is, but rather something else (if anything). A possible counterexample to this position is the following. Consider the holocaust. If the atrociousness of the holocaust were to be captured by the FA analysis and the amount of the disvalue is modeled by for how long it is fitting to feel contempt towards such a crime against humanity, then we will end up with a unacceptable conclusion. Say that it is fitting to feel contempt for 100 years for the holocaust. When the 100 years has passed it can clearly not be the case that it is no longer fitting not to feel contempt. The passage of time cannot have such an effect. This is just one consideration and we acknowledge that there is more to be said on the topic of temporal aspects of reasons and the FA analysis.

38 We believe that the notion of resilience is quite interesting. It might have some application when one, in an ever-changing world, is to choose between two things that are equally good, one should go for the object with the more resilient value. Another possibility is that one can be more certain that an object
} 
a brief characterization of what we take resilience to be. The value of $x$ can be more resilient than $y$ 's in the sense that $x$ value can withstand a greater shift in the balance of reasons and still remain valuable. To illustrate, assume that an object is valuable if and only if there are conclusive reason to favor it. Whether a reason is conclusive depends on the balance of reasons. If the balance of reasons were to shift so that we no longer have conclusive reason to favor the object then the object would no longer be valuable.

The more the balance of reasons can shift while it still being the case that we have conclusive reason to favor $x$ the more resilient the value of $x$ is. Thus, if there is a conclusive reason to favor $x$ to a certain degree of intensity, and a conclusive reason to favor $y$ to the very same degree of intensity, $x$ and $y$ are equally as valuable. Their respective value is determined by the attitudinal component. However, in our scenario let us assume that the pro tanto reasons to favor $x$ vastly outweighs the pro tanto reasons to not favor $x$, i.e., the balance of reasons is heavily in favor of favoring $x$. Furthermore, the pro tanto reasons to favor y only minimally outweigh the pro tanto reasons against favoring $y$. In our pictured scenario, $x$ 's value is more resilient. The value of $x$ is more resilient than $y$ 's value because it would take a greater shift in the balance of reasons to make it the case that we do not have conclusive reasons to favor $x$ than in the corresponding case of $y$.

With the concept of resilience we can account for the intuition that the normative component plays a role in determining the value of an object. It does not determine the degree of value, but rather another dimension of the object's value - its resilience.

\subsection{Value Comparatives and Monadic Values}

We have claimed that too little has been said in the literature about how the Fitting Attitudes analysis of value accounts for how valuable an object is. This should be interpreted to mean that little has been said about degrees of value, however, there are suggestions in the literature on how a proponent of the FA analysis should understand comparative value. More specifically there is a suggestion of how to analyze "better than" that seems to be widely endorsed in the FA analysis tradition: ${ }^{39}$

FA Better than: An object $x$ is better than $y$ iff it is fitting to prefer $x$ over $y$.

This analysis tells us how good $x$ and $y$ relative to each other without referring to the strength of the normative component or the degree of intensity of the attitudinal component. That is, we know that $x$ is better than $y$ without knowing whether it is more fitting to favor $x$ than it is fitting to favor $y$, or whether it is fitting to favor

Footnote 38 (continued)

will retain its value even if the object, or the world itself, were to change. Or that the notion of resilience allows us to separate choice-worthiness from value, say, providing a rationale for it being rational to choose being shot to death by one assassin rather than being shot to death by two assassins, shooting simultaneously. For more on the role of resilience see Henrik Andersson \& Jakob Green Werkmäster "The Resilience of Value" (MS).

39 See for instance Wlodek Rabinowicz, "Value relations revisited," Economics and Philosophy 28(2) (2012): 133-164 and Schroeder, op. cit. 
$x$ with a higher degree of intensity than $y$. Prima facie we seem to have an analysis that is neutral with regards to the Normative Component View and the Attitudinal Component View. However, we are not given any information about $x$ 's and $y$ 's monadic value. It might be that both are bad, but that $x$ is less bad than $y$. In other words, we are given an account of value comparisons without expressing anything about the compared objects' monadic values. This is of course a possibility but it seems odd to have an analysis that can account for how valuable the object is in relation to other objects without accounting for how valuable the object is by itself, i.e., the analysis is silent on whether the object is very good, somewhat good, or even bad. This line of reasoning is familiar from debates on whether "better than" is more basic than "good." 40 Note, however, that the aforementioned objection is even more problematic in this context since the aim of this text is to provide an analysis of how good something is. Providing an answer to how valuable $x$ is (i.e., accounting for its monadic value) in terms of FA "better than" is only a serious contender if it is possible to understand "good" in terms of "better than." The prospects of this has recently, however, be shown to be quite slim by Johan Gustafsson. ${ }^{41}$

Let us clarify what it means for the comparative analysis to be neutral with regards to the two views under consideration. First, it can be concluded that this view is compatible with both the Normative Component View and the Attitudinal Component View. According to the Normative Component View if $x$ is better than $y$ then it must be more fitting to favor $x$ than it is fitting to favor $y$. This may very well be compatible with the fact that it is fitting to prefer $x$ over $y$. There is no contradiction in these claims. According to the Attitudinal Component View, if $x$ is better than $y$ then it is fitting to favor $x$ with a stronger intensity than $y$. This as well seems to be compatible with it being fitting to prefer $x$ over $y$.

Even though there are no contradictions between these views, it is not obvious how this comparative formulation of the FA analysis relates to the two views discussed here. Can this account be derived from the strength of the normative component and/ or the intensity of the attitudinal component that is directed to the objects in isolation as is suggested in the above accounts? Or is it a completely separate account?

Let us first consider what the possibilities are for the Attitudinal Component View. Consider the claim that how valuable the object is should be understood in terms of the degree of intensity of the attitude it is fitting to have towards the object. It may be a conceptual necessity that if it is fitting to favor $x$ with a certain intensity and it is fitting to favor $y$ less intense, then it is fitting to prefer $x$ over $y$. After all, if it is fitting to favor $x$ with a certain intensity and it is fitting to favor $y$ less intense, then clearly it is fitting to favor $x$ more strongly than $y$. And to favor something more strongly seems to entail preferring it. Or at least the more moderate claim seems true: If one prefers $x$ over $y$ in terms of admirations, then one admires $x$ more than one admires $y$.

\footnotetext{
40 See John Broome, Ethics Out of Economics. (Cambridge: Cambridge University Press, 1999), Erik Carlson, "Good' in Terms of 'Better'," Noûs, 50(1) (2016): 213-223, and Johan Gustafsson, "Neither 'Good' in Terms of 'Better' nor 'Better' in Terms of 'Good',' Nô̂s, 48(1), (2014): 466-473.

41 See Gustafsson, op. cit.
} 
Of course, "favoring" is here taken as a placeholder for any pro-attitude. The reasoning in the previous paragraph, however, assumes that there is a corresponding comparative attitude to all monadic pro-attitudes. But one can question whether all comparative value have a comparative attitude. On the other hand, it seems to be the case that all attitudes with intensity can have a comparative aspect. As long as the attitude is more intense it seems to follow that one can express a comparison using this attitude. Consequently, the Attitudinal Component View seems to provide us with an answer to why "better than" can be analyzed in terms of fitting preferences.

We can provide an outline of an argument that attempts to show the connection between the Normative Component View and the above analysis of "better than." In order to argue that the FA analysis of value comparatives is derivable from the Normative Component View, one seems forced to argue that it is the very fact that is more fitting to favor $x$ than it is fitting to favor $y$ that makes it fitting to prefer $x$ to $y$. In other words, one is committed to the following principle:

P1 Whenever it is more fitting to favor one object than it is fitting to favor another, it will also be fitting to prefer the former to the latter.

$P 1$, however, is in tension with the following:

P2 If it is fitting to prefer $x$ over $y$ in terms of admirability, then it is fitting to admire $x$ more intensely than $y$.

$P 2$ seems to follow from a reasonable view on the logic of preferences. If one prefers $x$ over $y$ then ones desire to $x$ is stronger than ones desire to $y$. Which entails that if one prefers $x$ over $y$ in terms of admirability then one admires $x$ more intensely than $y$. From this we can assume that if it is fitting to prefer $x$ over $y$ in terms of admirability, then it is fitting to admire $x$ more intensely than $y$.

Consider the following example to see why $P 1$ and $P 2$ are not compatible. Imagine that it is very fitting to admire Ann with a low intensity and merely fitting to admire Beth with a high intensity. From $P l$ it follows that it will be fitting to prefer Ann to Beth in terms of admirability. However, according to $P 2$ this entails that it is fitting to admire Ann more intensely than Beth. This seems implausible considering that it is very fitting to admire Ann with a low intensity and merely fitting to admire Beth with a high intensity. ${ }^{42}$

To conclude; FA analysis of comparatives does not seem to be as straightforwardly derivable from the Normative Component View on its own. This could of course be taken as a reason to reject the Normative Component View. The fact that the most accepted version of how one ought to analyze value comparatives is not straightforwardly derivable from the Normative Component View should count for

\footnotetext{
42 It could be objected that this implausible result will not be the case if we accept the following: If the normative component for admiring is stronger for $x$ than for $y$, then the attitudinal component to admire is more intense for $x$ than for $y$. But this seems rather ad hoc and should rather be described as a kind of hybrid view.
} 
something. ${ }^{43}$ Furthermore, both the Attitudinal Component View and the Normative Component View would turn out to entail that if $x$ is better than $y$ in terms of admiration then it is fitting to admire $x$ more intensely than $y$. The difference is that the Attitudinal Component View arrives at this conclusion more naturally than the Normative Component View, where it is perhaps at most viewed as an unfortunate consequence. The simplicity of the Attitudinal Component view seems to speak somewhat in favor of it over the Normative Component View. The FA analysis of "better than" consequently seems to be compatible with the Normative Component View and the Attitudinal Component View, but only derivable from the latter.

\section{Conclusions}

We have distinguished some possible positions on how the FA analysis can account for the amount of value an object has. Implications of the various positions have been drawn and their different merits discussed. Since this is an undertheorized but highly interesting topic, we put more emphasis on outlining the various positions and their implications. We believe that our findings show that the Normative Component View is not as promising as the Attitudinal Component View; in other words, if an object is very valuable it is fitting to favor it with a high degree of intensity. This approach is more in line with previous work within the FA analysis tradition and faces fewer theoretical limitations and hurdles. Another up-shot of the paper is the notion of resilience of an object's value. With the help of resilience we can provide a plausible account of the intuition that the normative component should make some difference with regard to how valuable the object.

Acknowledgements Open access funding provided by Lund University. We are grateful for the valuable feedback we have received on earlier versions of this paper. We are especially grateful to David Alm, Andrés Garcia, Mattias Gunnemyr, Marta Johansson, Jonas Olson Wlodek Rabinowicz, Andrew Reisner, and Toni Rønnow-Rasmussen. A version of this paper was presented at The Future of Normativity conference, University of Kent (2018) we are thankful for the valuable comments we received at that occasion. Henrik Andersson's research was funded by Åke Wibergs foundation and Birgit och Gad Rausings foundation.

Open Access This article is licensed under a Creative Commons Attribution 4.0 International License, which permits use, sharing, adaptation, distribution and reproduction in any medium or format, as long as you give appropriate credit to the original author(s) and the source, provide a link to the Creative Commons licence, and indicate if changes were made. The images or other third party material in this article are included in the article's Creative Commons licence, unless indicated otherwise in a credit line to the material. If material is not included in the article's Creative Commons licence and your intended use is not permitted by statutory regulation or exceeds the permitted use, you will need to obtain permission directly from the copyright holder. To view a copy of this licence, visit http://creativecommons.org/licenses/by/4.0/.

Publisher's Note Springer Nature remains neutral with regard to jurisdictional claims in published maps and institutional affiliations.

\footnotetext{
43 To this one could object that it merely points to the possibility that we should reject the suggested FA analysis of "better than". This is of course a possibility, but the suggested FA analysis of "better than" has the advantage of allowing for a bigger conceptual space for value relations, in fact it allows room for as much as 15 value relations according to Rabinowicz 2008 op. cit,. This theoretical virtue talks in favor or analyzing value relations in the manner suggested.
} 